\title{
L-CARNITINE SUPPLEMENTATION DECREASES DNA DAMAGE IN TREATED MSUD PATIENTS
}

Caroline Paula Mescka1,2; Gilian Guerreiro ${ }^{1,3}$; Bruna Donida ${ }^{1,2}$; Desirèe Marchetti ${ }^{1,2}$, Carlos Eduardo Jacques $^{1,2}$; Moacir Wajner ${ }^{1,2}$, Carlos Severo Dutra-Filho ${ }^{2}$, Carmen Regla Vargas S $^{1,2,3}$

${ }^{1}$ Serviço de Genética Médica, HCPA, UFRGS, Porto Alegre, RS, Brazil; ${ }^{2}$ Programa de PósGraduação em Ciências Biológicas:Bioquímica, UFRGS, Porto Alegre, RS, Brazil; $\quad{ }^{3}$ Programa de Pós-Graduação em Ciências Farmacêuticas, UFRGS, Porto Alegre, RS, Brazil;

Introduction: Maple syrup urine disease (MSUD) is an inherited disorder of branchedchain amino acids (BCAA). Patients generally present psychomotor delay, mental retardation and brain abnormalites. Studies have shown that oxidative stress may be involved in neuropathology of MSUD. In this regard, it was recently reported that MSUD patients have L-carnitine (L-car) deficiency, a compound with antioxidant properties that is used as adjuvant therapy in some inborn errors of metabolism. Objective: we evaluated the role of dietary L-car supplementation on DNA damage in whole blood leukocytes from MSUD patients and the relationship between DNA lesions, malondialdehyde (MDA) levels and L-car concentrations in plasma from MSUD patients. Material and methods: Six MSUD patients admitted at the Hospital de Clinicas de Porto Alegre (mean age $8.28 \pm$ 2.87 years) were supplemented with a BCAA-restricted diet and L-car (50 mg kg-1 day-1). The DNA damage (determined by the alkaline comet assay), as well as MDA, a marker of lipid peroxidation, and L-car concentrations were determined in blood of MSUD patients before, after 1 month and 2 months of L-car supplementation and compared to control group (six age-matched healthy individuals (mean age $6.0 \pm 3.12$ years)), using repeated measures of ANOVA, followed by the Tukey multiple range test). Results: We observed a significant increase of DNA damage index (DI) in leukocytes from MSUD patients under BCAA-restricted diet as compared to controls and L-car supplementation significantly decreased DNA DI levels $(P<0.05)$. It was also found a positive correlation between DI and MDA content $(r=0.93 ; p<0.05)$, and an inverse correlation between DI and L-car levels $(r=-0.82 ; p<0.05)$. Conclusion: Our results suggest the involvement of oxidative stress in DNA damage in this disorder. Since L-car reduced DNA damage, it is presumed that dietary supplementation of this compound may serve as an adjuvant therapeutic strategy for MSUD patients. The study was approved by the Ethics Committee of HCPA (project 140191). Financial support: FAPERGS, CNPq and FIPE/HCPA-Brazil. E-mail of presenter: carolmescka@yahoo.com.br 